\title{
MicroRNA-543 suppresses colorectal cancer growth and metastasis by targeting KRAS, MTA1 and HMGA2
}

\author{
Chuannan Fan ${ }^{1, *}$, Yancheng Lin ${ }^{1, *}$, Yubin Mao ${ }^{1,2, *}$, Zhengjie Huang ${ }^{3, *}$, Allan Yi Liu ${ }^{1}$, \\ Handong $\mathrm{Ma}^{1}$, Donghong $\mathrm{Yu}^{1}$, Alaiyi Maitikabilii ${ }^{1}$, Hongjun Xiao ${ }^{1}$, Chuankai Zhang ${ }^{3}$, \\ Fan Liu², Qi Luo ${ }^{3}$, Gaoliang Ouyang ${ }^{1,4}$ \\ ${ }^{1}$ State Key Laboratory of Cellular Stress Biology, Innovation Center for Cell Signaling Network, School of Life Sciences, \\ Xiamen University, Xiamen, China \\ ${ }^{2}$ Medical College, Xiamen University, Xiamen, China \\ ${ }^{3}$ Department of Surgical Oncology, First Affiliated Hospital of Xiamen University, Xiamen, China \\ ${ }^{4}$ Engineering Research Centre of Molecular Diagnostics, Ministry of Education, School of Life Sciences, Xiamen University, \\ Xiamen, China \\ *These authors contributed equally to this work
}

Correspondence to: Gaoliang Ouyang, e-mail: oygldz@xmu.edu.cn

Keywords: miR-543, colorectal cancer, metastasis, proliferation, microRNA

Received: September 23, 2015

Accepted: February 23, 2016

Published: March 08, 2016

\section{ABSTRACT}

miR-543 has been implicated as having a critical role in the development of breast cancer, endometrial cancer and hepatocellular carcinoma. However, the exact clinical significance and biological functions of miR-543 in colorectal cancer (CRC) remain unclear. Here, we found that miR-543 expression significantly downregulated in tumors from patients with CRC, APCMin mice and a mouse model of colitis-associated colon cancer. miR-543 level was inversely correlated with the metastatic status of patients with CRC and the metastatic potential of CRC cell lines. Moreover, ectopic expression of miR-543 inhibited the proliferation and metastasis of CRC cells in vitro and in vivo by targeting KRAS, MTA1 and HMGA2. Conversely, miR-543 knockdown promoted the proliferation, migration and invasion of CRC cells in vitro and augmented tumor growth and metastasis in vivo. Furthermore, we found that miR-543 expression was negatively correlated with the levels of KRAS, MTA1 and HMGA2 in clinical samples. Collectively, these data show that miR-543 inhibits the proliferation and metastasis of CRC cells by targeting KRAS, MTA1 and HMGA2. Our study highlights a pivotal role for miR-543 as a suppressor in the regulation of CRC growth and metastasis and suggests that miR-543 may serve as a novel diagnostic and prognostic biomarker for CRC metastasis.

\section{INTRODUCTION}

As one of the most common malignant cancers worldwide, colorectal cancer (CRC) has become the fifth leading cause of cancer death for men and women in China [1]. Distant metastasis accounts for the majority of cancer-related mortality in patients with $\mathrm{CRC}$, and the 5 -year survival rate for metastatic CRC is only $10-15 \%$ [2-5]. Therefore, a better understanding of the molecular mechanisms involved in CRC metastasis will provide diagnostic and prognostic markers and potential targets for the therapeutic intervention of CRC metastasis. It has been demonstrated that several key molecular events, including mutations in KRAS, aberrant activation of the Wnt and PI3K pathways and TP53 inactivation, are involved in the initiation and progression of CRC [6-9]. In addition to genetic aberrances, epigenetic alterations such as changes in microRNA (miRNA) deregulation and chromatin structure contribute to the development of CRC [10]. miRNAs are endogenous noncoding regulatory RNAs that inhibit gene expression at the post-transcriptional level by binding to the $3^{\prime}$-untranslated-region (3'UTR) of target mRNAs [11,12]. Although great advances have been achieved in elucidating the specific roles of miRNAs in the initiation, progression and metastasis of CRC, the underlying mechanisms remain largely elusive $[5,10,13]$. 
As a member of a miRNA cluster located in the imprinted DLK1-DIO3 region on human chromosome 14, miR-543 has been reported to function as a tumor suppressor in breast cancer and endometrial cancer [14, 15], whereas it was found to promote the tumorigenesis of hepatocellular carcinoma [16]. However, none of the previous studies has systematically investigated the functions of the miR-543 in the progression of CRC. In this study, we evaluated the clinical significance and biological functions of miR-543 using clinical CRC samples, tissues from two mouse CRC models, and a panel of CRC cell lines with different metastatic potentials. We demonstrate that miR-543 inhibits the growth and metastasis of CRC cells in vitro and in vivo by targeting KRAS, MTA1 and HMGA2. Our study suggests that miR-543 may be a critical determinant of CRC progression.

\section{RESULTS}

\section{miR-543 expression is downregulated in CRC tissues and inversely correlated with CRC metastasis}

miR-543 has been described as a tumor suppressor gene for breast cancer and endometrial cancer $[14,15]$ but as an oncogene for hepatocellular carcinoma [16]. To investigate the clinicopathological significance of miR-543 in CRC, we first detected the expression of miR-543 in 45 paired human CRC tissues and matched normal colorectal tissues. As shown in Figure 1A, the level of miR-543 was decreased in 34 of the 45 (75.6\%) CRC tissues compared with the normal counterparts. We found that miR-543 expression was reduced by nearly 3-fold in the CRC tissues compared with their corresponding nontumorous colorectal tissues (median 5.8 and 15.7, respectively; $p<0.001$ ) (Figure 1B). Clinicopathologic analysis revealed that the expression of miR-543 was also negatively correlated with distant metastasis status (Figure 1C) and N classification (Table 1); however, no significant difference was observed between the level of miR-543 and sex, age or T classification of patients with CRC (Table 1). We further determined the level of miR-543 in highly metastatic human CRC cell lines (SW620 and LoVo) and CRC cell lines with low metastatic potential (HCT116, LS174T, HT29 and Caco-2). The level of miR-543 was relatively lower in highly metastatic CRC cell lines than those in the four tumorigenic but low-metastatic cell lines (Figure 1D), indicating that miR-543 level is inversely correlated with the metastatic potential of CRC cell lines.

To further evaluate the role of miR-543 in CRC progression, we used two mouse CRC models, $\mathrm{APC}^{\mathrm{Min}}$ mice and azoxymethane/dextran sodium sulfate (AOM/ DSS) mice. The $\mathrm{APC}^{\mathrm{Min}}$ mouse model is a spontaneous CRC model whereas AOM/DSS model is a colitisassociated CRC model [17-19]. Mice in both models formed numerous visible tumors in colorectal epithelium. Using qRT-PCR analysis, we found that the level of
miR-543 in CRC tumors isolated from APC Min mice was significantly lower than that in colorectal epithelium tissues from wild-type mice (Figure 1E). Similarly, mice treated with AOM/DSS showed a significantly decreased level of miR-543 in CRC tissues compared with that in colorectal epithelium tissues in the control group (Figure 1F). Taken together, these data demonstrate that miR-543 expression is reduced in clinical CRC specimens and mouse CRC tissues, and its level is inversely correlated with the metastatic potential of CRC cell lines and the metastatic status of patients with CRC.

\section{KRAS, MTA1 and HMGA2 are direct targets of miR-543}

To explore the tumor-suppressive roles of miR-543 in $\mathrm{CRC}$, we further examined the putative downstream targets of miR-543 by three in silico prediction algorithms (miRanda, TargetScan and miRWalk). Several in silico prediction algorithm-identified oncogenes including KRAS, MTA1, HMGA2, ADAM9, FMNL2 and SIRT1, which contain putative binding sites for miR-543 in their 3'UTRs, were chosen for further investigation. First, we cloned 3'UTRs that contain putative miR-543 binding sites into the pmiR report luciferase construct, and each was co-transfected with a miR-543 expression plasmid into HEK293T and SW620 cells. Dual-luciferase reporter assays revealed that the luciferase activities of KRAS, MTA1 and HMGA2 but not FMNL2, SIRT1 or ADAM9 significantly decreased in both HEK293T (Figure 2A) and SW620 cells (Figure 2B) upon miR-543 overexpression. However, the inhibitory effects were abolished when the putative miR543 seed-binding regions in the 3'UTRs of KRAS, MTA1 and HMGA2 were mutated (Figure 2C and 2D). These data demonstrate that KRAS, MTA1 and HMGA2 are direct targets of miR-543.

\section{miR-543 inhibits CRC cell proliferation in vitro}

To evaluate the role of miR-543 in CRC progression, we first determined the effects of miR-543 overexpression on the biological functions of human CRC cell lines. Two highly metastatic CRC cell lines, SW620 and LoVo, which have very low endogenous miR-543 expression, were stably infected with a lentivirus expressing miR-543 (Supplementary Figure S1). MTT assays revealed that ectopic overexpression of miR-543 in SW620 and LoVo cells resulted in a significant decrease in cell proliferation compared with control cells (Figure 3A and 3B). Moreover, the overexpression of miR-543 significantly decreased the colony numbers of SW620 and LoVo cells (Figure 3C and 3D). The KRAS-RAF-MEK-ERK-Cyclin D1 signaling pathway is a key promoter of the CRC malignant process through its effects on cell proliferation $[20,21]$; thus, we next determined whether miR-543 inhibits CRC cell proliferation by targeting KRAS. As shown in Figure 3E, overexpression of miR-543 in SW620 and LoVo cells 
decreased the expression of KRAS and Cyclin D1 at the mRNA level. Western Blot analysis further revealed that miR-543 overexpression decreased the expression of KRAS and Cyclin D1 and the phosphorylation level of MEK (p-MEK) and ERK (p-ERK) compared with controls (Figure 3F). Together, these results indicate that miR-543 may inhibit the proliferation of CRC cells by downregulating the KRAS-related oncogenic pathway.

\section{miR-543 suppresses CRC cell migration and invasion in vitro}

We next examined whether miR-543 could suppress the motility and invasiveness of CRC cells by targeting MTA1 and HMGA2. Transwell assays showed that overexpressing of miR-543 in SW620 and LoVo cells significantly inhibited cell migration (Figure 4A) and
A

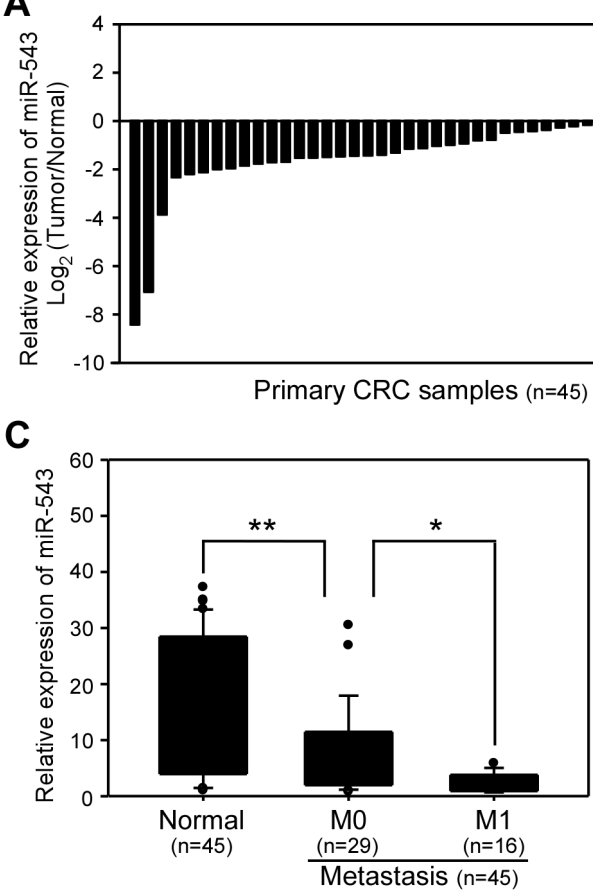

$\mathbf{E}$
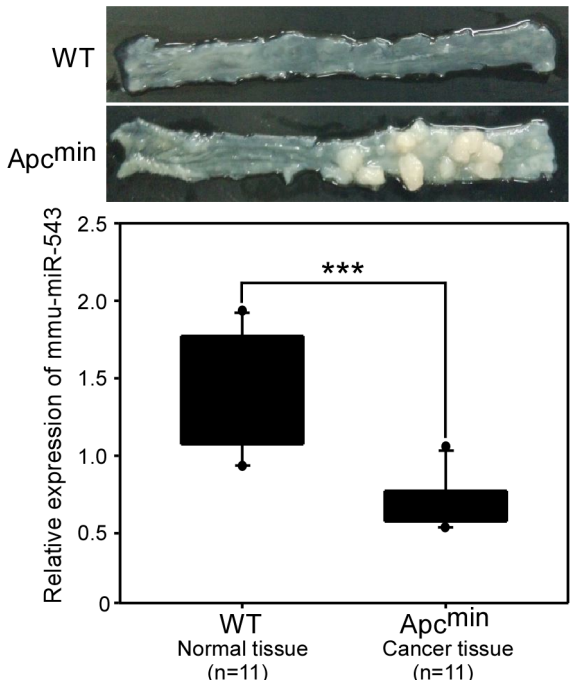

B

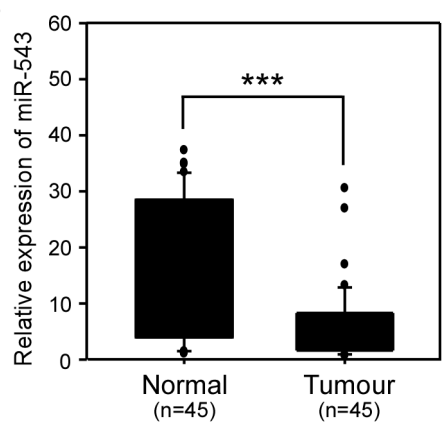

D

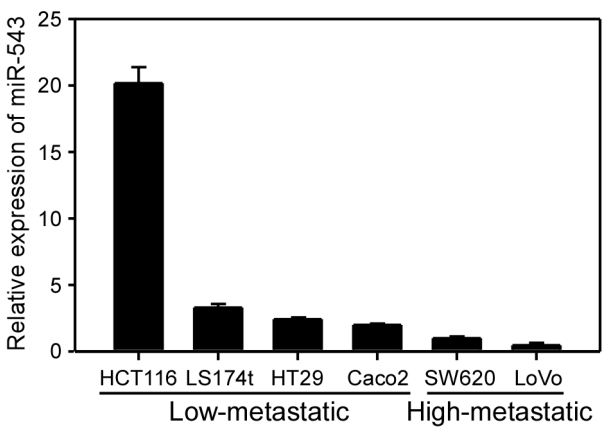

$\mathbf{F}$

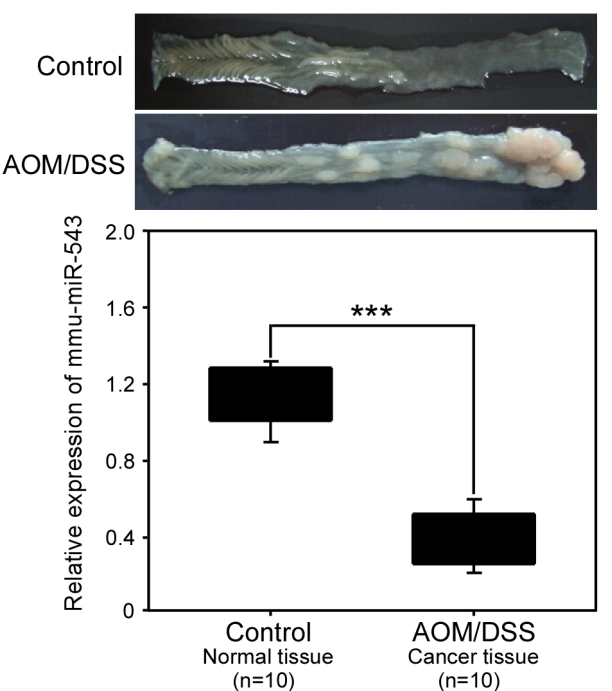

Figure 1: miR-543 expression is downregulated in clinical colorectal cancer (CRC) samples, CRC cell lines and mouse CRC tissues. (A, B) qRT-PCR analysis of miR-543 expression in human CRC tissues and matched normal colon tissues from 45 patients with CRC. Data were expressed as $\log 2$ fold change (relative miR-543 expression in tumor sample/relative miR-543 expression in matched normal colon tissue) to show the relative expression in every paired samples (A) and the relative expression difference between all normal colon samples and tumor samples (B). (C) Correlation between miR-543 expression and the distant metastasis status of CRC. (D) qRT-PCR analysis of miR-543 expression in CRC cell lines with different metastatic potentials. (E, F) Representative pictures of colon tissues (top) and qRT-PCR analysis of mmu-miR-543 expression (bottom) in wild-type (WT) and Apc ${ }^{\text {Min }}$ mice $(n=11)(\mathbf{E})$, and in control and AOM/ DSS-treated mice $(n=10)(\mathbf{F}) * p<0.05, * * p<0.01, * * * p<0.001$. 
Table 1: Correlation of relative miR-543 expression with the clinicopathological characteristics of patients with colorectal cancer

\begin{tabular}{|c|c|c|c|c|}
\hline \multirow{2}{*}{ Clinicopathological feature } & \multirow{2}{*}{ Number of cases } & \multicolumn{3}{|c|}{ miR-543 levels* } \\
\hline & & High & Low & $P$ Value \\
\hline \multicolumn{5}{|l|}{ Age (years) } \\
\hline$>61$ & 25 & 15 & 10 & \multirow{2}{*}{0.12} \\
\hline$\leq 61$ & 20 & 6 & 14 & \\
\hline \multicolumn{5}{|l|}{ Gender } \\
\hline Male & 31 & 16 & 15 & \multirow{2}{*}{0.13} \\
\hline Female & 14 & 5 & 9 & \\
\hline \multicolumn{5}{|l|}{ T classification } \\
\hline I-II & 19 & 7 & 12 & \multirow{2}{*}{0.082} \\
\hline III-IV & 26 & 8 & 18 & \\
\hline \multicolumn{5}{|l|}{$\mathrm{N}$ classification } \\
\hline N0 & 17 & 12 & 5 & \multirow{2}{*}{0.009} \\
\hline $\mathrm{N} 1-\mathrm{N} 2$ & 28 & 11 & 17 & \\
\hline \multicolumn{5}{|l|}{ Distant metastasis } \\
\hline No & 29 & 11 & 18 & \multirow{2}{*}{0.030} \\
\hline Yes & 16 & 4 & 12 & \\
\hline
\end{tabular}

*The mean (5.8) of the relative expression of miR-543 in tumor tissues of all paired samples was used as a cut-off to classify a tumor sample was High or Low according to their miR-543 expression levels.

invasion (Figure 4B) in vitro. MTA1 and HMGA2 have been reported to promote EMT and the acquisition of metastatic potential for CRC epithelial cells [22, 23]; thus, we next determined whether the suppression of the migration and invasion of CRC cells by miR-543 was an EMT-related behavior. Upon ectopic overexpression of miR-543, we did not observe significant alterations of the epithelial cell markers E-cadherin and $\beta$-catenin or the mesenchymal cell markers N-cadherin and Vimentin in SW620 and LoVo cells (Supplementary Figure S2), indicating that the miR-543related inhibition of cell migration and invasion is dependent on mechanisms other than EMT. MTA1 has been reported to promote lung metastasis of breast cancer by stimulating STAT3 transcription and the expression of STAT3 target genes [24]. The secretion of MMPs contributes to the abnormalities of the tumor microenvironment during tumor metastasis [25]. We found that miR-543 overexpression in CRC cells decreased the mRNA and protein levels of MTA1 and STAT3 and the mRNA level of their downstream genes $M M P 2$ and MMP9 (Figure 4C and 4D). In addition, we also observed that ectopic expression of miR-543 reduced the levels of pro- and cleaved- MMP2 and MMP9 in
SW620 and LoVo cells by using gelatin zymography assay (Supplementary Figure S3). HMGA2 can promote breast cancer cell invasion by remodeling the extracellular matrix (ECM) [26]. Here, we found that miR-543 overexpression in SW620 and LoVo cells increased the level of miR-200b but decreased the expression of HMGA2 and LOX at the mRNA (Figure 4C) and protein levels (Figure 4D). Moreover, the phosphorylation level of p-FAK, a downstream effector of LOX [27], was also decreased upon miR-543 overexpression (Figure 4D). These data indicate that miR-543 suppresses CRC invasion in vitro by targeting MTA1 and HMGA2.

Next, we performed rescue experiments to further confirm that miR-543 inhibits the malignant phenotypic alterations of CRC cells by directly repressing the three target genes. To abrogate the suppression of miR-543 on KRAS, MTA1 and HMGA2, plasmids expressing each target gene lacking a $3^{\prime}$ UTR were constructed and transduced into LoVo-miR-543 cells (Figure 4E). Strikingly, exogenous expression of these three target genes almost completely rescued the miR-543-induced inhibitory effects on cell proliferation (Figure 4F) and colony formation (Supplementary Figure S4). Moreover, restoration of 
KRAS, MTA1 or HMGA2 in CRC cells at least partially reversed the inhibitory effects on cell migration imposed by miR-543 expression (Figure 4G; Supplementary Figure S5). Therefore, re-expression of KRAS, MTA1 or HMGA2 reverses the miR-543-induced inhibition of the proliferation, migration and invasion of CRC cells in vitro, indicating that KRAS, MTA1 and HMGA2 are functional targets of miR-543 in CRC cells.

\section{miR-543 overexpression inhibits tumor growth and metastasis of CRC cells in vivo}

After determining that miR-543 overexpression significantly suppresses the proliferation, migration and invasion of CRC cells in vitro, we further investigated whether miR-543 could inhibit the growth and metastasis of CRC cells in vivo. SW620 and LoVo cells that stably overexpress miR-543 or their control cells were infected with a virus encoding the luciferase gene and then subcutaneously injected into nude mice and analyzed after 4 weeks. Bioluminescence imaging results showed that mice injected with SW620-Ctrl cells formed larger tumors than those bearing miR-543-overexpressing CRC cells (Figure 5A). We next isolated xenograft tumors and confirmed that the overexpression of miR-543 in SW620 cells significantly decreased subcutaneous tumor growth compared with the control groups, and the volumes and weights of the tumors of the nude mice injected with miR-543-overexpressing
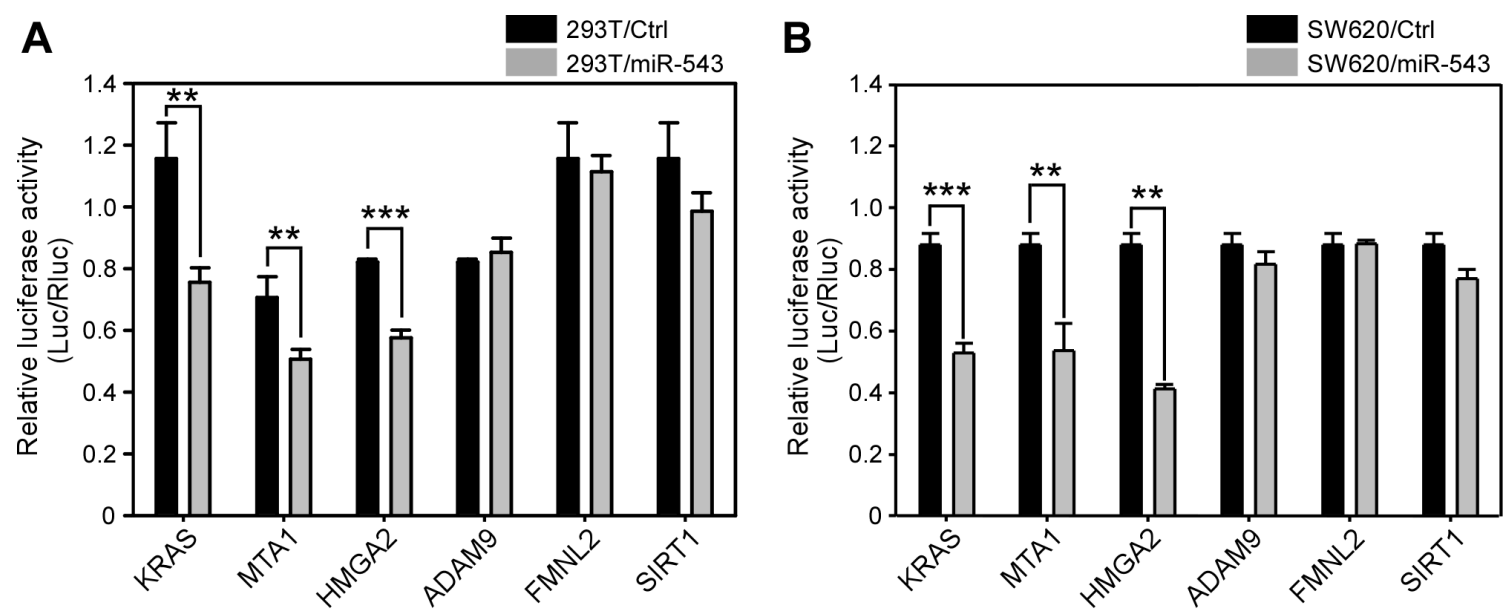

C $\begin{array}{lc}\text { KRAS-WT 5'-GUUGCGCUGACCUAGGAAUGUUG-3' } & \\ \text { miR-543 } & \text { 3'-UUCUUCACGUGGCGCUUACAAA-5' } \\ \text { KRAS-Mut } & \text { 5'-GUUGCGCUGACCUAGGATUAAUG-3' }\end{array}$

$\begin{array}{lc}\text { MTA1-WT } & \text { 5'-UGUAACUUACACCUGGAAUGUUA-3' } \\ \text { miR-543 } & \text { 3'-UUCUUCACGUGGCGCUUACAAA-5' } \\ \text { MTA1-Mut } & \text { 5'-UGUAACUUACACCUGGATCAUUA-3' }\end{array}$

$\begin{array}{lc}\text { HMGA2-WT } & \text { 5'-CUCAAUACUACCUCUGAAUGUUA-3' } \\ \text { miR-543 } & \text { 3'-UUCUUCACGUGGCGCUUACAAA-5' } \\ \text { HMGA2-Mut } & \text { 5'-CUCAAUACUACCUCUGTTCGUUA-3' }\end{array}$

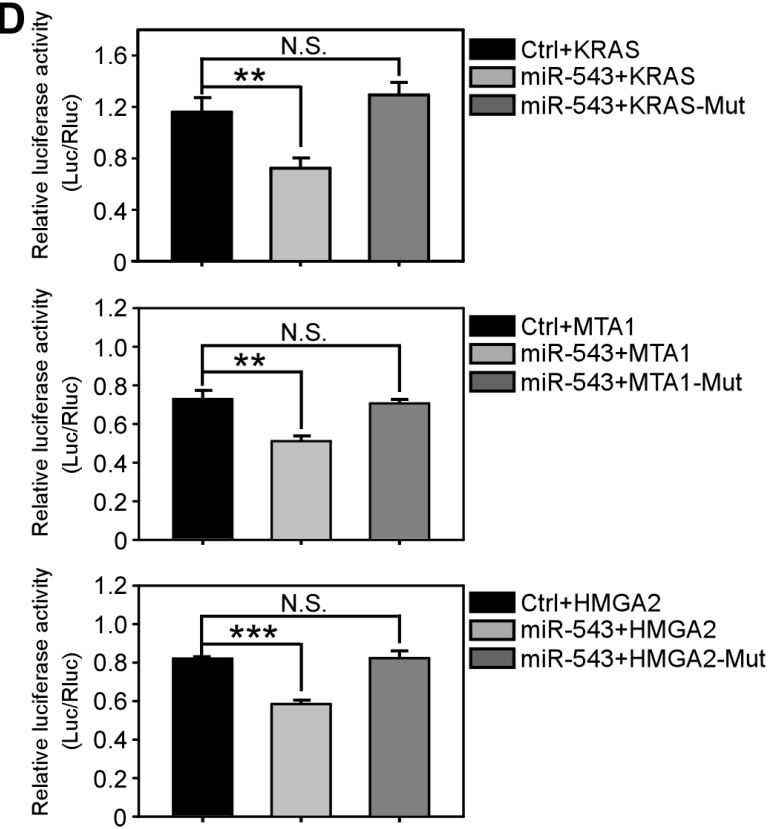

Figure 2: KRAS, MTA1 and HMGA2 are downstream targets of miR-543. (A, B) Dual luciferase reporter assay analysis of the effects of miR-543 overexpression on the activities of 3'UTRs of predicted target genes in 293T (A) and SW620 cells (B). (C) Mutations were generated in the 3'UTR sequences of the KRAS, MTA1 and HMGA2 mRNAs at the complementary sites for the seed regions in miR-543. (D) Dual luciferase reporter assay analysis of the effects of miR-543 expression on the activities of the wild-type and mutant 3'UTRs of KRAS, MTA1 and HMGA2 in 293T cells. These results are representative of at least three independent experiments. ** $p<0.01$, $* * * p<0.001$, N.S: no significance. 


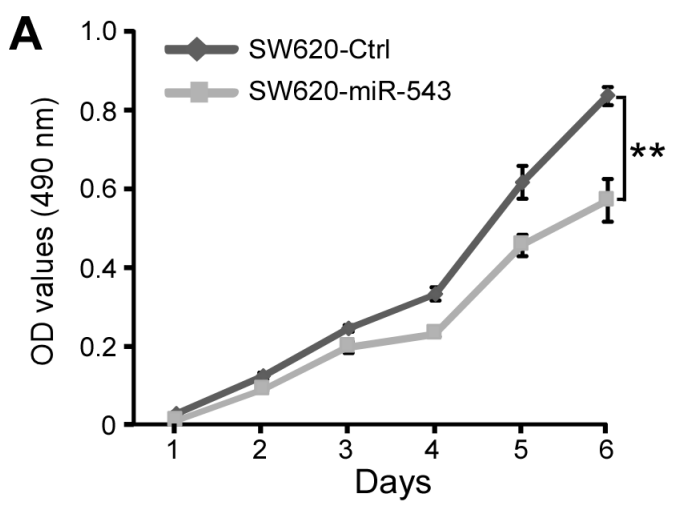

C

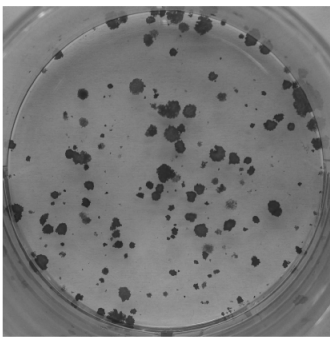

SW620-Ctrl

D

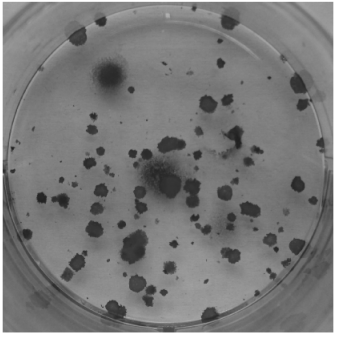

LoVo-Ctrl

E
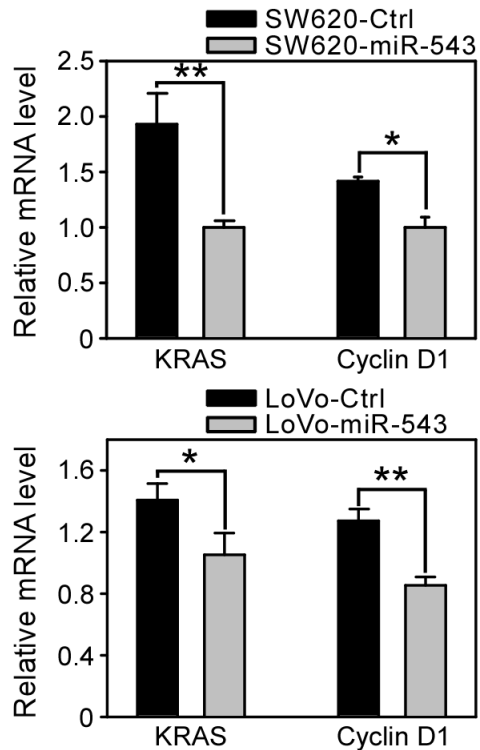

$\mathbf{F}$
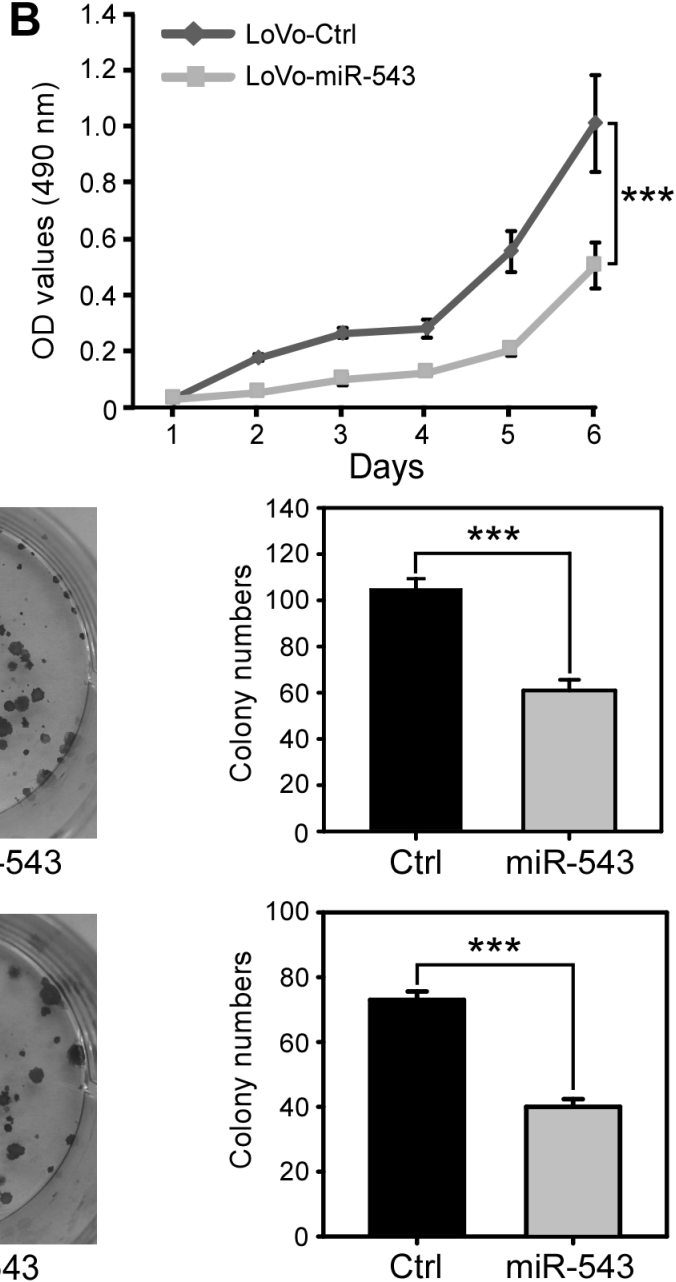

LoVo-miR-543
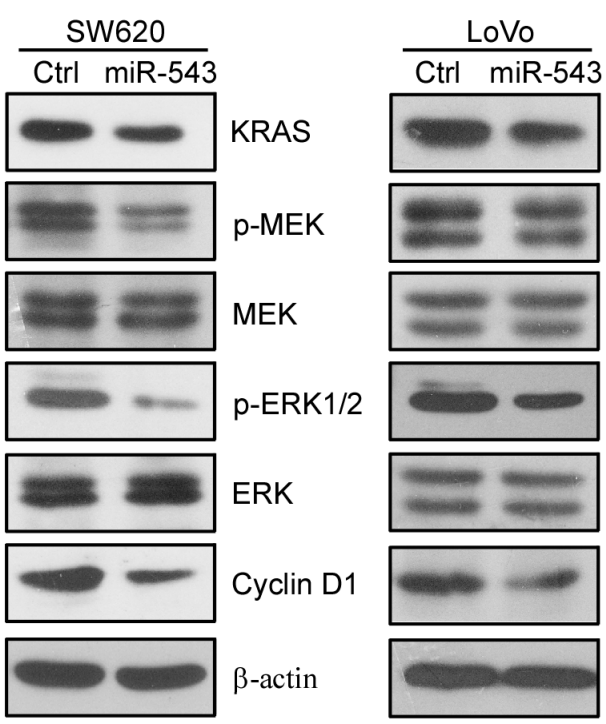

KRAS

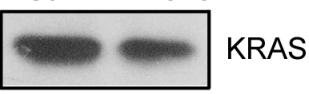

-MEK

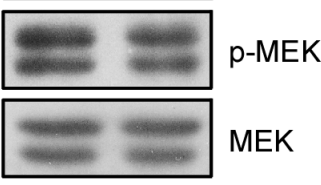

-ERK1/2

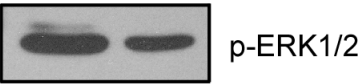

ERK

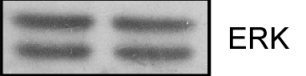

Cyclin D1

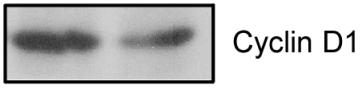

$\beta$-actin

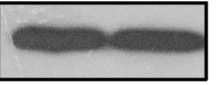

$\beta$-actin

Figure 3: miR-543 overexpression inhibits the proliferation of CRC cells in vitro. (A, B) MTT analysis of the effects of miR543 on the proliferation of SW620-Ctrl and SW620-miR-543 (A) and LoVo-Ctrl and LoVo-miR-543 cells (B). (C) Representative images and quantification of the colonies formed by SW620-Ctrl and SW620-miR-543 cells. (D) Representative images and quantification of colonies formed by LoVo-Ctrl and LoVo-miR-543 cells. (E) qRT-PCR analysis of the mRNA levels of KRAS and Cyclin D1 in SW620Ctrl, SW620-miR-543, LoVo-Ctrl and LoVo-miR-543 cells. (F) Western blot analysis of the levels of KRAS and proliferation-related proteins p-MEK, MEK, p-ERK1/2, ERK and Cyclin D1 in SW620-Ctrl, SW620-miR-543, LoVo-Ctrl and LoVo-miR-543 cells. These results are representative of at least three independent experiments. ${ }^{*} p<0.05, * * p<0.01, * * * p<0.001$. 
A

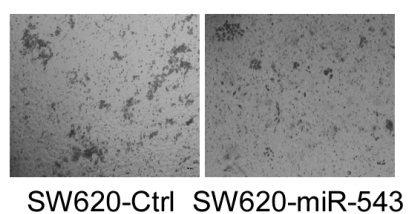

B
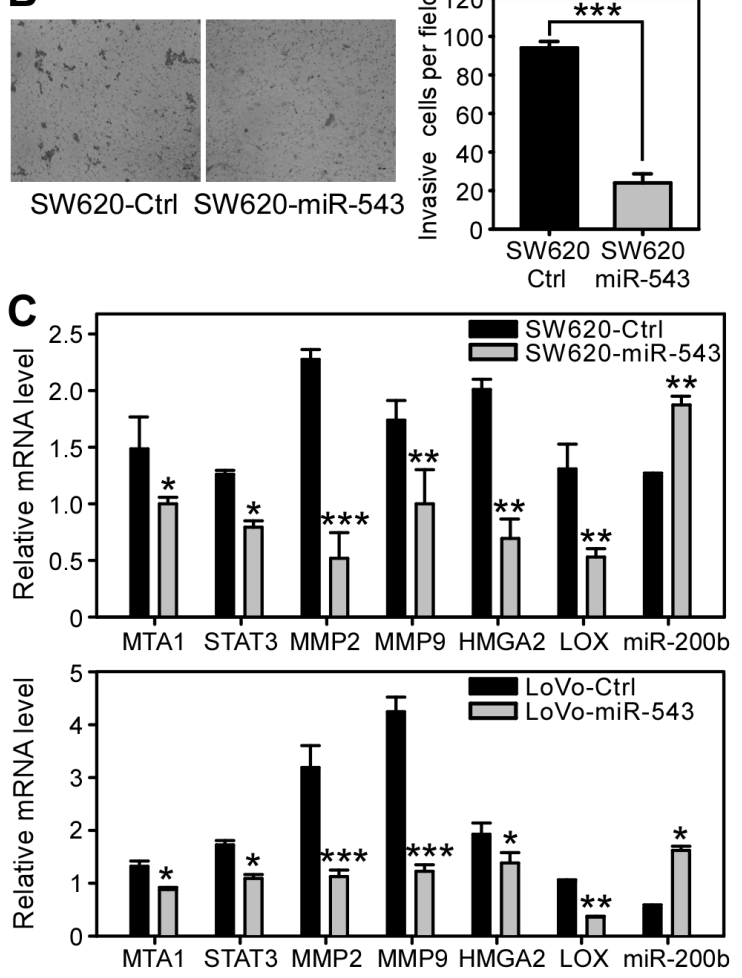

E

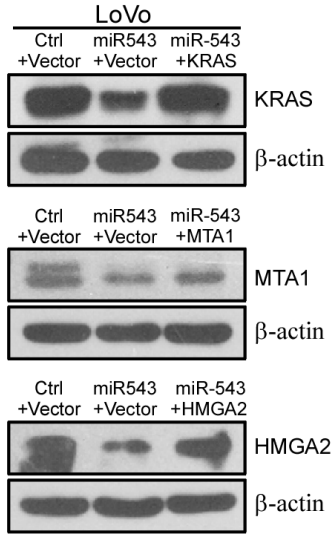

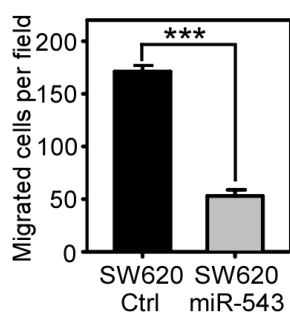

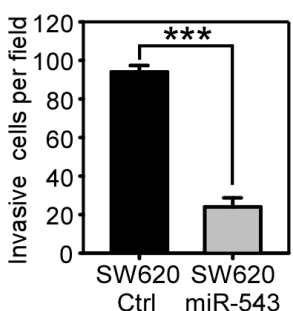

$\mathbf{F}$

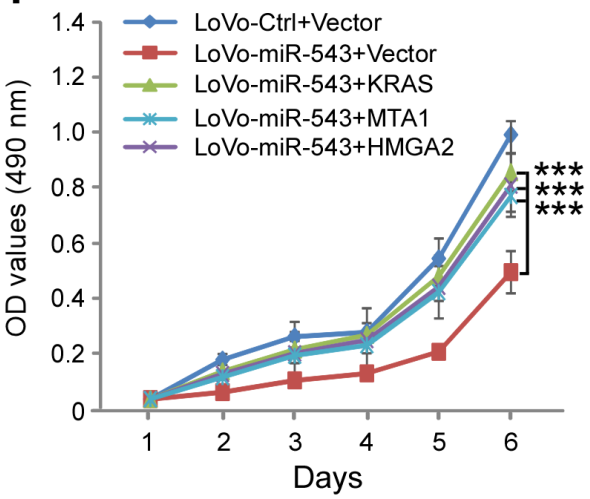

LoVo-Ctrl

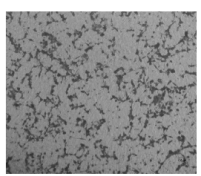

LoVo-Ctrl

D

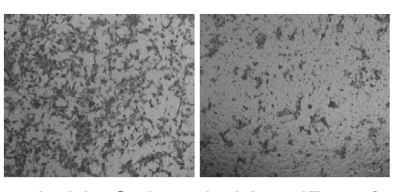

LoVo-miR-543
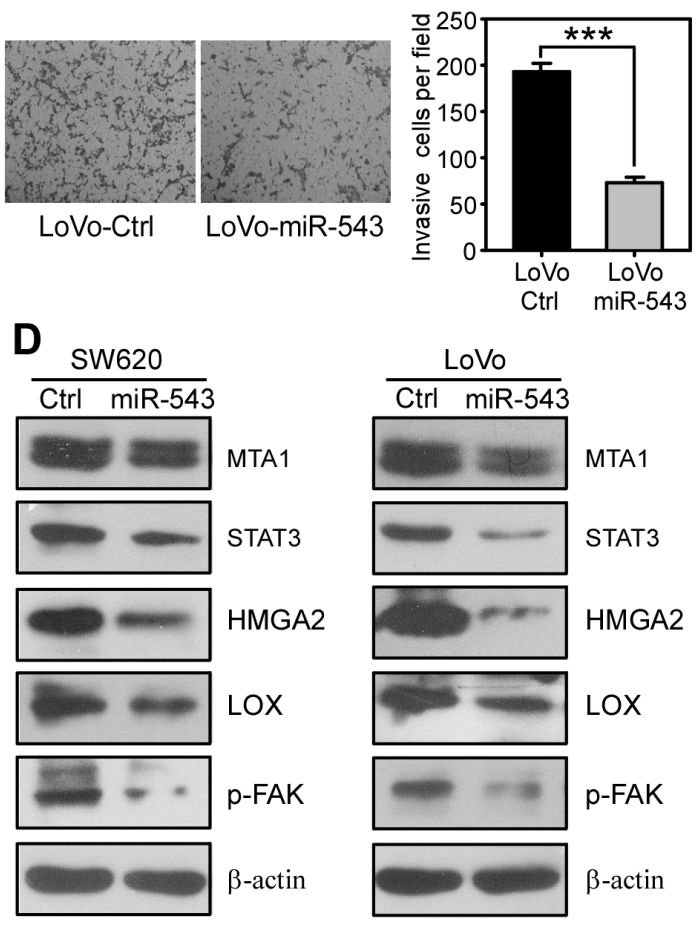

G

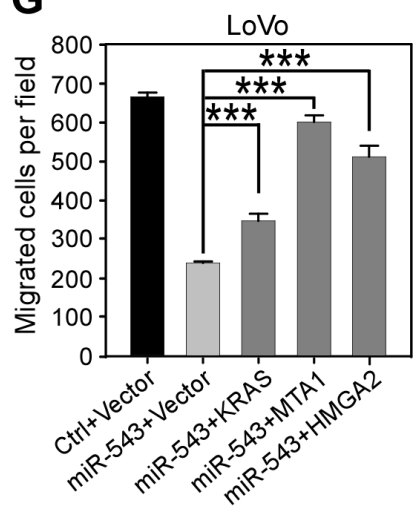

Figure 4: miR-543 overexpression suppresses the migration and invasion of CRC cells in vitro and re-expression of KRAS, MTA1 and HMGA2 reverses the miR-543-induced effects on CRC cells. (A) Transwell migration assays revealed that ectopic overexpression of miR-543 inhibits the migration ability of SW620 and LoVo cells. (B) Transwell invasion assay with Matrigel-coated membranes revealed that miR-543 overexpression inhibits the invasion ability of SW620 and LoVo cells. (C) qRT-PCR analysis revealed that miR-543 downregulates the mRNA expression of MTA1 and HMGA2 and their downstream genes STAT3, MMP2, MMP9 and LOX but upregulates miR-200b in SW620 (top) and LoVo (bottom) cells. (D) Western blot analysis of the effects of miR-543 overexpression on the expression of MTA1 and HMGA2 and their downstream targets STAT3, LOX and p-FAK in SW620 (left) and LoVo (right) cells. (E) Western blot analysis of KRAS, MTA1 or HMGA2 re-expression in LoVo-miR-543 cells. LoVo-miR-543 cells were transfected with miRNA-resistant expression constructs for KRAS, MTA1 or HMGA2. Control (Ctrl) represents scrambled miRNA, and vector represents the empty vector used in KRAS, MTA1 and HMGA2 re-expression. (F, G) MTT assay $(\mathbf{F})$ and migration $(\mathbf{G})$ analysis of the proliferation of LoVo-miR-543 cells transfected with miRNA-resistant expression constructs for KRAS, MTA1 or HMGA2. These results are representative of at least three independent experiments. Scale bars, $100 \mu \mathrm{m} .{ }^{*} p<0.05,{ }^{* *} p<0.01, * * * p<0.001$. 
cells decreased compared with those of the control group (Figure 5A). Similarly, stable expression of miR-543 inhibited the tumor growth of LoVo cells in vivo (Figure 5B). Moreover, tumors from miR-543-overexpressing cells had lower Ki67 expression compared with tumors from controls (Figure 5C). These data indicate that miR-543 inhibits the tumor growth of CRC cells in vivo.

Distant spread of CRC cells to the intestines and liver and the formation of macroscopic metastases are the major cause of CRC mortality [28, 29]. We next evaluated whether miR-543 could inhibit the ability of CRC cells to metastasize to the intestines and liver. SW620, LoVo and their miR-543-overexpressing cells, which also expressed the luciferase gene, were intrasplenically injected into nude mice to develop experimental intestinal and hepatic metastasis. As shown in Figure 5D, bioluminescence imaging revealed that miR-543 overexpression significantly reduced the metastasis of SW620 cells in nude mice. We isolated several organs that were reported to have higher CRC metastatic tropism [30] and found that mice bearing miR-543-overexpressing SW620 cells had less intestinal and liver metastases than control mice (Figure 5D; Supplementary Figure S6A). Similar inhibitory effects of miR-543 on tumor metastasis were observed in the mice bearing LoVo-Ctrl or LoVomiR-543 cells (Figure 5E; Supplementary Figure S6B). Collectively, these observations demonstrate that miR-543 inhibits the tumor growth and metastasis of CRC cells in vivo.

\section{Knockdown of miR-543 promotes the proliferation, invasion and metastasis of CRC cells in vitro and in vivo}

We further performed loss-of-function experiments to verify the function of miR-543 in CRC cells. To this end, we stably knocked down the expression of miR-543 in HCT116 cells, which has relatively high endogenous miR-543 expression, using a lentivirus-based antagomir expression system (Supplementary Figure S7) [31]. As shown in Figure $6 \mathrm{~A}$ and $6 \mathrm{~B}$, miR-543 knockdown led to a significant increase in cell proliferation and colony number. Consistent with these phenotypes, the mRNA levels of KRAS and Cyclin D1 and the protein levels of KRAS, p-MEK, p-ERK and Cyclin D1 upregulated when endogenous miR-543 was suppressed (Figure 6C and 6D). Moreover, in agreement with this result, knockdown of endogenous miR-543 increased the mRNA expression of MTA1, HMGA2, STAT3, MMP2, MMP9 and LOX but decreased the level of miR-200b. The protein levels of MTA1 and HMGA2 and their downstream effectors STAT3, LOX and p-FAK were dramatically upregulated (Figure 6C and 6D). Furthermore, the suppression of endogenous miR-543 dramatically promoted the migration (Figure 6E) and invasion (Figure 6F) of HCT116 cells. However, this proinvasive phenotype was irrelevant to the EMT program
(Supplementary Figure S8). Thus, the loss-of-function data demonstrate that miR-543 knockdown promotes the proliferation, migration and invasion of CRC cells in vitro.

We further investigated whether miR-543 knockdown could promote the growth and metastasis of CRC cells in vivo. HCT116 cells that stably knockdown miR-543 or their control cells were subcutaneously injected into nude mice for 4 weeks and analyzed by bioluminescence imaging. As shown in Figure 6G, mice injected with HCT116-Ctrl cells formed smaller tumors than those mice bearing miR543-knockdown CRC cells. Moreover, the metastatic ability of HCT116 cells significantly increased when endogenous miR-543 was knocked down (Figure 6H). These in vivo data indicate that knockdown of miR-543 markedly promoted the tumor growth and metastasis of CRC cells.

\section{Inverse correlation between miR-543 and its targets in clinical CRC samples}

To extend our findings to human CRC, we examined whether the miR-543-related suppression of KRAS, MTA1 and HMGA2 in CRC cells is clinically relevant. We used two paired CRC samples and their matched nontumorous tissues to detect the protein expression of KRAS, MTA1 and HMGA2 by immunohistochemical staining. Relative miR-543 expression $(\mathrm{T} / \mathrm{N})$ was 0.86 in one paired samples and this paired samples was considered to be a case with high miR-543 expression, whereas another paired samples whose relative miR-543 expression was 0.30 was regarded as a case with low miR-543 expression. Quantitative immunohistochemical staining results revealed that CRC tissues with a high level of miR-543 had low expression of KRAS, MTA1 and HMGA2, whereas CRC tissues with a low level of miR-543 exhibited high expression of KRAS, MTA1 and HMGA2, indicating that miR-543 expression is inversely correlated with the levels of KRAS, MTA1 and HMGA2 in clinical CRC tissues (Figure 7).

\section{DISCUSSION}

It has been reported that miR-543 is downregulated in breast cancer [14] and endometrial cancer [15] but functions as an oncogene in hepatocellular carcinoma [16]. In this study, we report that the expression of miR-543 is significantly downregulated in human and mouse CRC tissues and is inversely correlated with the $\mathrm{N}$ classification and metastatic status of patients with CRC. Both our in vitro and in vivo results support that miR-543 significantly inhibits the growth and metastasis of CRC. These findings demonstrate that miR-543 may function as a tumor suppressor or oncogene in a context-dependent manner; therefore, the discrepancy that miR-543 exhibits opposing effects in different tumors deserves further investigation.

Using in silico analysis and dual-luciferase reporter assays, we identified KRAS, MTA1 and HMGA2 as three new direct miR-543 downstream targets. KRAS is 

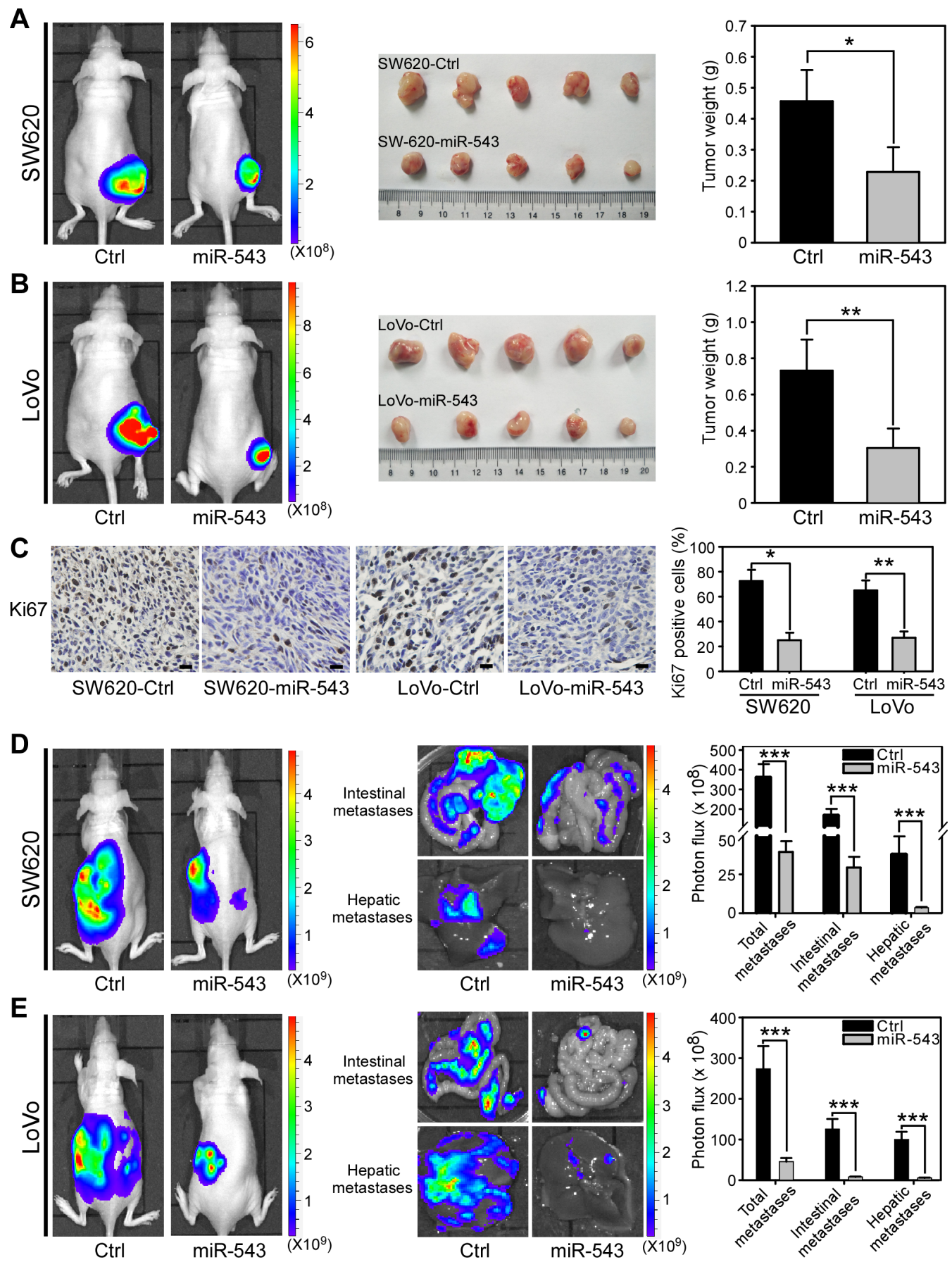

Figure 5: miR-543 overexpression inhibits the tumor growth and metastasis of CRC cells in vivo. (A) Bioluminescence images of nude mice showing tumors derived 28 days after subcutaneous injection in the lower back regions of nude mice with SW620Ctrl or SW620-miR-543 cells (left). Images of isolated tumors (middle) and quantitation of tumor weights (right) from mice 28 days after injection with SW620-Ctrl or SW620-miR-543 cells. $n=5$ per group. (B) Bioluminescence images of nude mice showing tumors derived 28 days after subcutaneous injection in the lower back regions of nude mice with LoVo-Ctrl or LoVo-miR-543 cells (left). Images of isolated tumors (middle) and quantitation of tumor weights (right) from mice 28 days after injection with LoVo-Ctrl or LoVo-miR-543 cells. $n=5$ per group. (C) IHC staining analyses and quantitation of Ki-67-positive tumor cells in xenograft tumors from mice 28 days after injection with SW620-Ctrl, SW620-miR-543, LoVo-Ctrl or LoVo-miR-543 cells. (D) Bioluminescence images of metastatic tumors in mice 28 days after intrasplenic injection with SW620-Ctrl or SW620-miR-543 cells (left). Bioluminescence images of intestines and livers isolated from mice 28 days after intrasplenic injection with SW620-Ctrl or SW620-miR-543 cells (middle). Quantitation of metastases in the whole bodies, intestines and livers of these mice (right). $n=5$ per group. (E) Bioluminescence images of the metastatic tumors in mice 28 days after intrasplenic injection with LoVo-Ctrl or LoVo-miR-543 cells (left). Bioluminescence images of intestines and livers (left) isolated from mice 28 days after intrasplenic injection with LoVo-Ctrl or LoVo-miR-543 cells (middle). Quantitation of metastases in the whole bodies, intestines and livers of these mice (right). $n=5$ per group. Scale bars, $50 \mu \mathrm{m} . * p<0.05, * * p<0.01, * * * p<0.001$. 

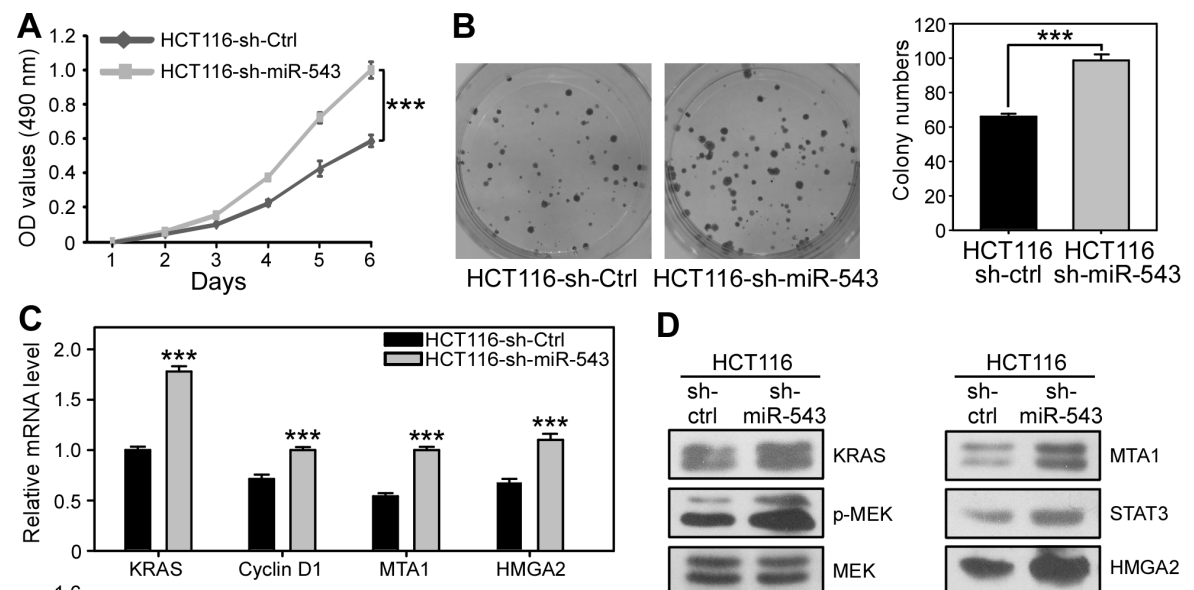

D
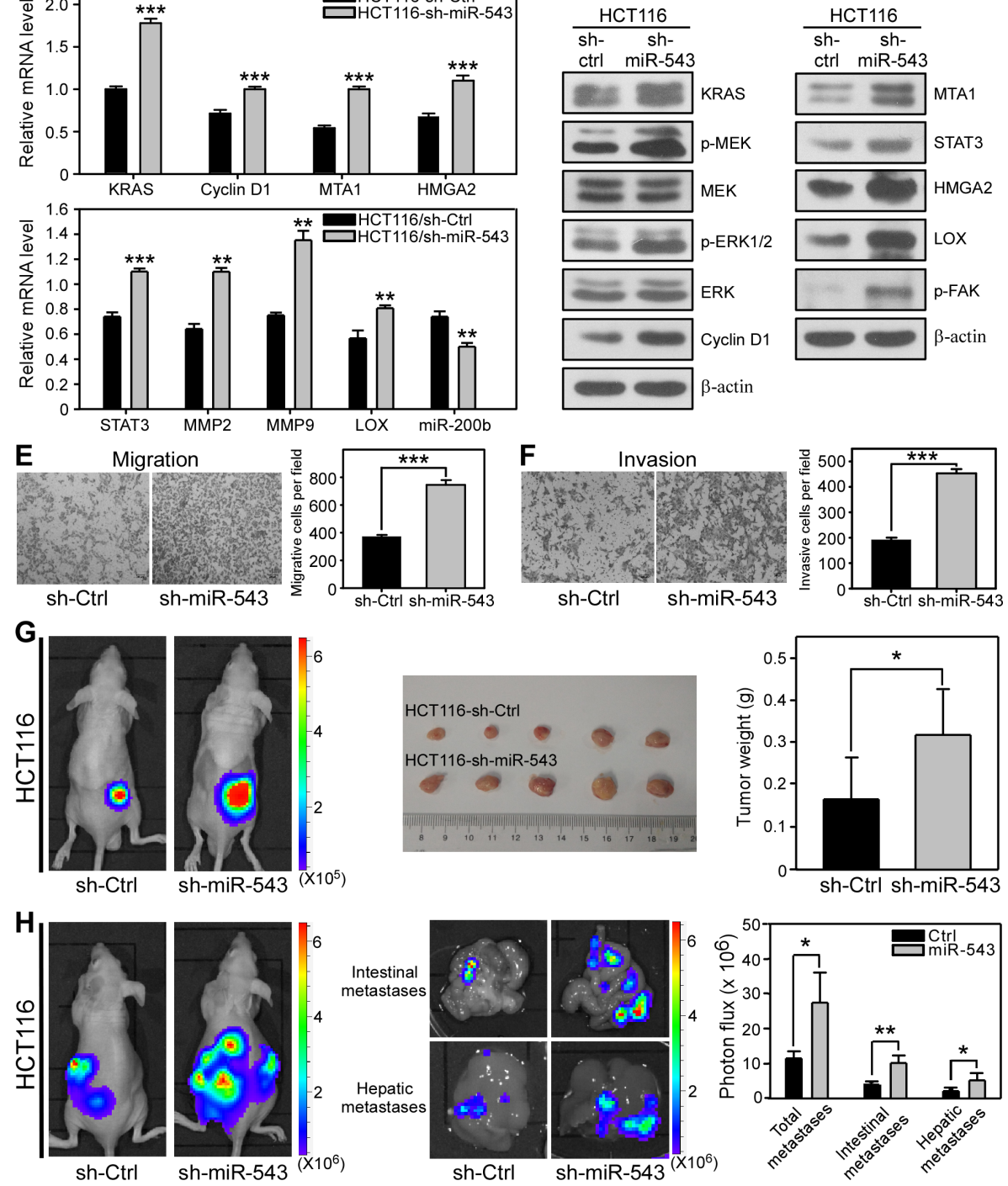

Figure 6: miR-543 knockdown promotes the proliferation, invasion and metastasis of HCT116 cells in vitro and in vivo. (A) MTT analysis of the effects of miR-543 on the proliferation of HCT116 cells. (B) Representative images and quantification of colonies formed by HCT116-sh-Ctrl and HCT116-sh-miR-543 cells. (C) qRT-PCR analysis revealed that miR-543 knockdown upregulates the mRNA expression of KRAS, MTA1 and HMGA2 and their downstream genes Cyclin D1, STAT3, MMP2, MMP9 and LOX but downregulates miR-200b in HCT116 cells. (D) Western blot analysis of the levels of KRAS and the proliferation-related proteins p-MEK, MEK, p-ERK1/2, ERK and Cyclin D1 (left), and MTA1 and HMGA2 and their downstream genes STAT3, LOX and p-FAK (right) in HCT116-sh-Ctrl and HCT116-sh-miR-543 cells. (E, F) Transwell migration assays (E) and Matrigel-coated membrane Transwell invasion assays (F) demonstrated that miR-543 knockdown promotes the migration and invasion of HCT116 cells in vitro. (G) Bioluminescence images of nude mice showing tumors derived 28 days after subcutaneous injection in the lower back regions of nude mice with HCT116sh-Ctrl or HCT116-sh-miR-543 cells (left). Images of isolated tumors (middle) and quantitation of tumor weights (right) from mice 28 days after injection with HCT116-sh-Ctrl or HCT116-sh-miR-543 cells. $n=5$ per group. (H) Bioluminescence images of metastatic tumors in mice 28 days after intrasplenic injection with HCT116-sh-Ctrl or HCT116-sh-miR-543 cells (left). Bioluminescence images of intestines and livers isolated from mice 28 days after intrasplenic injection with HCT116-sh-Ctrl or HCT116-sh-miR-543 cells (middle). Quantitation of metastases in the whole bodies, intestines and livers of these mice (right). $n=5$ per group. Scale bars, $200 \mu \mathrm{m} .{ }^{*} p<0.05,{ }^{* *} p<0.01$, $* * * p<0.001$. 
an oncogene that was found to be mutationally activated in approximately half of early-stage CRCs [32]. MTA1 is a critical regulator of the metastatic process in various types of cancers including CRC [22, 33]. MTA1 enhances lung metastasis of breast cancer by upregulating STAT3 transcription [24], which promotes cell survival, angiogenesis, invasion and metastasis [34, 35]. HMGA2 dysregulation is correlated with poor survival for patients with CRC [36] and promotes breast cancer metastasis by increasing LOX expression [26]. Here, our data reveal that there is an inverse correlation between the miR-543 level and the expression of its targets, KRAS, MTA1 and HMGA2, in clinical CRC samples. Moreover, miR-543 overexpression inhibits the growth and metastasis of CRC cells in vitro and in vivo by targeting KRAS, MTA1 and HMGA2. Conversely, knockdown of miR-543 promotes the proliferation, invasion and metastasis of CRC cells in vitro and in vivo. Therefore, these results support the notion that the downregulation of miR-543 in CRC cells "contributes to the activation of multiple oncogenic signaling" pathways by upregulating the expression of KRAS, MTA1 and HMGA2, and ultimately results in $\mathrm{CRC}$ progression and metastasis.

Amplifications, deletions or mutations of miRNA loci, epigenetic silencing and transcriptional dysregulation are common mechanisms that lead to the aberrant expression of specific miRNAs [37]. We demonstrated that miR-543 is downregulated in human CRC samples, mouse CRC tissues and CRC cell lines with highly metastatic potential. However, the underlying mechanisms that regulate miR-543 expression remain elusive. In ductal carcinoma samples, $\mathrm{CpG}$ islands on the upstream of primary-miR-543 have been shown to be hypermethylated, which directly results in the down-regulation of mature miR-543 [14]. Thus, further investigations are needed to determine whether this epigenetic mechanism or other events are causal factors for the downregulation of miR-543 in CRC progression.
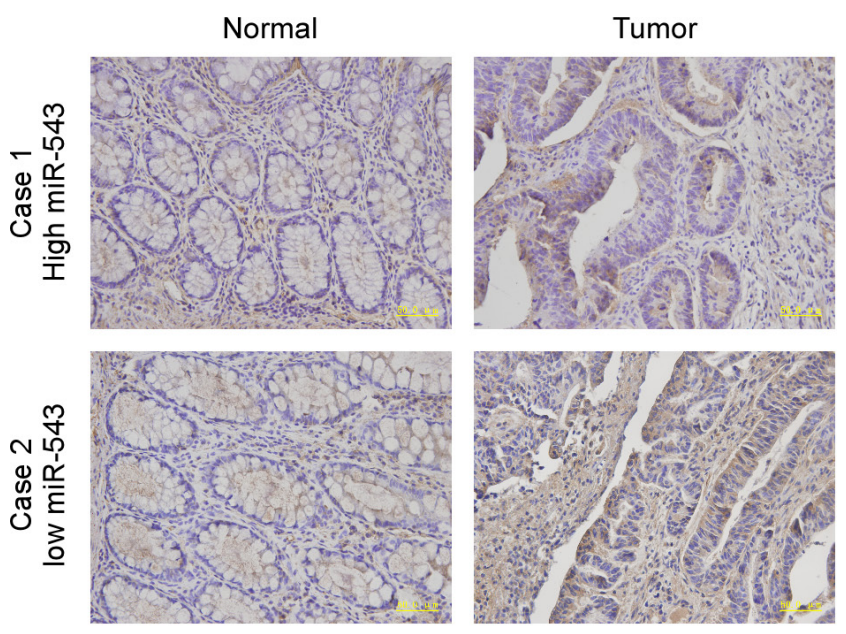

KRAS

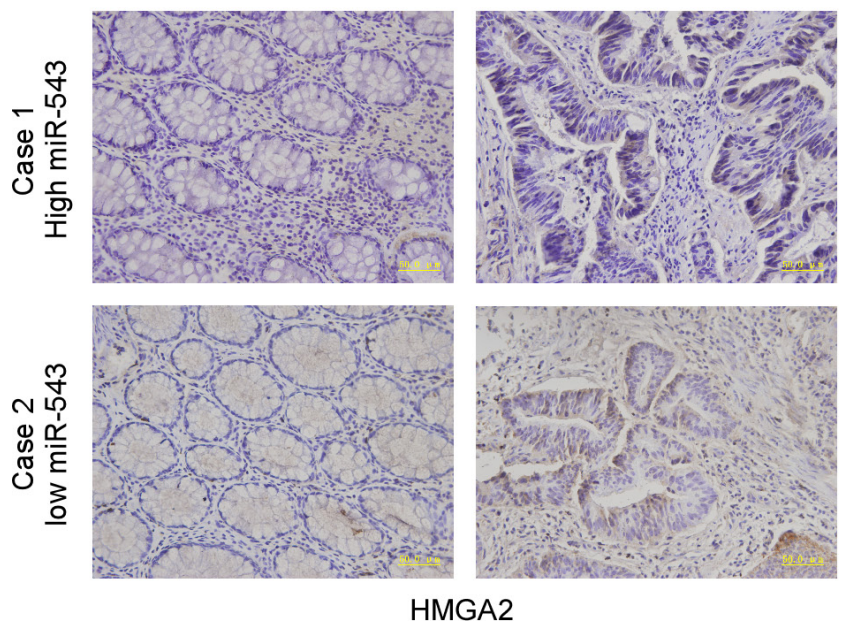

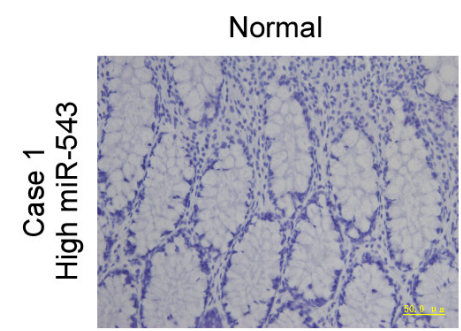
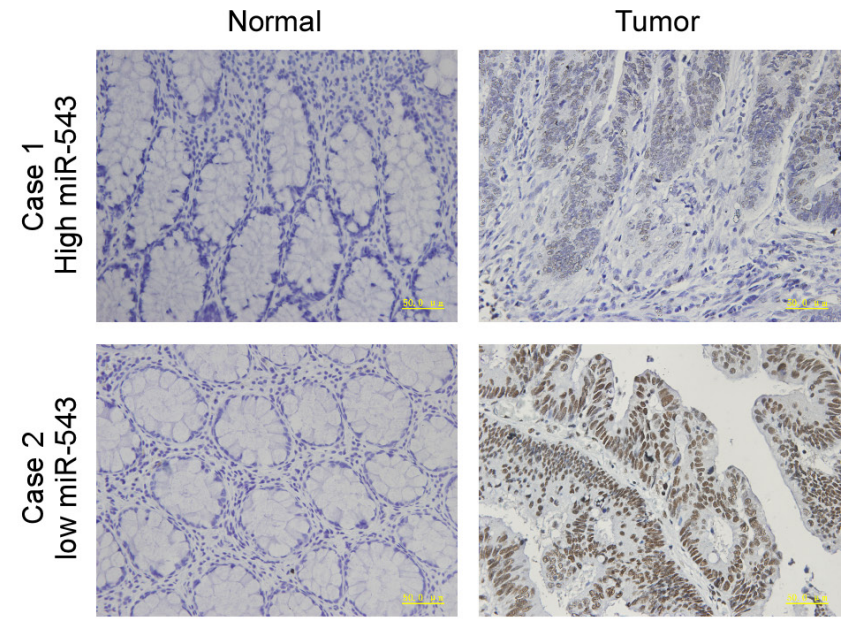

MTA1

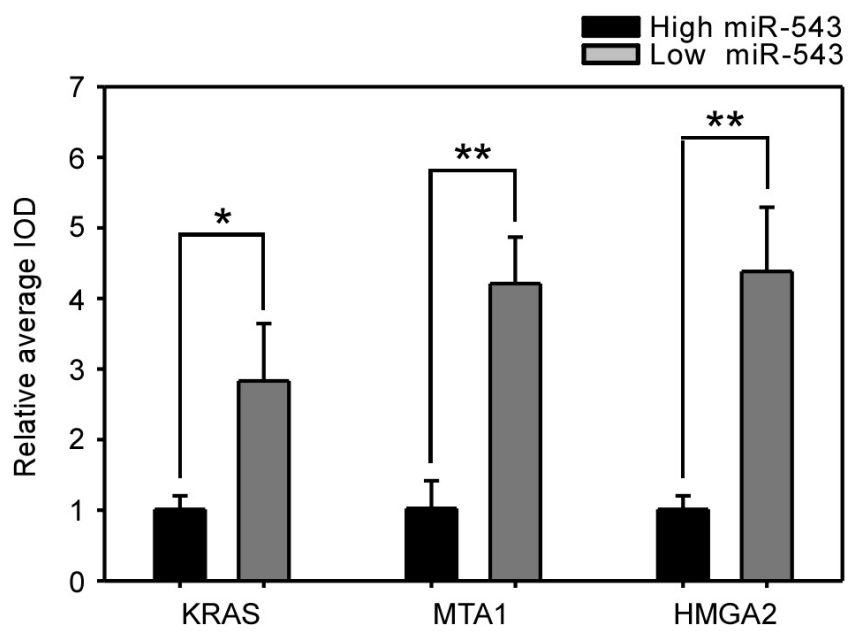

Figure 7: Clinical correlation analysis of the level of miR-543 and its targets in CRC tissues. Representative immunohistochemical staining images of the expression of KRAS, MTA1 and HMGA2 in two paired primary CRC tissues with low or high level of miR-543 and analyses of relative average integrated optical density (IOD) using Image Pro-Plus software. Scale bars, $50 \mu \mathrm{m}$. $* p<0.05, * * p<0.01$. 
In conclusion, our study highlights a pivotal role for miR-543 as a tumor suppressor in the regulation of CRC cell proliferation and metastasis by targeting KRAS, MTA1 and HMGA2 and suggests that miR-543 may serve as a novel diagnostic and prognostic biomarker for CRC metastasis.

\section{MATERIALS AND METHODS}

\section{Clinical specimens}

For the use of clinical samples for research purposes, prior approval was obtained from the First Affiliated Hospital of Xiamen University. The institutional ethics and scientific committee approved this study. All of the CRC tumor tissues and matched adjacent normal tissues $(n=45)$ from patients with CRC were collected at the Department of Surgical Oncology, First Affiliated Hospital of Xiamen University. The matched clinical information was collected and analyzed with prior written informed consent from the patients. All the tissue biopsies were freshly frozen in liquid nitrogen and stored at $-80^{\circ} \mathrm{C}$ until further use. The medical records of the patients were reviewed to collect the following clinicopathological information: age, gender, metastasis and the clinical stage according to their $\mathrm{N}$ classification and $\mathrm{T}$ classification. The tumor, node, metastasis (TNM) staging system of the American Joint Committee on Cancer (AJCC) and the International Union Against Cancer (UICC) was used to classify the stage of clinical samples.

\section{Animal studies}

All of the experiments using animals were performed in accordance with a protocol approved by the Animal Care and Use Committee of Xiamen University. $\mathrm{Apc}^{\mathrm{Min}}$ mice in the C57BL/6 background were provided by Dr Jiahuai Han (Xiamen University, Xiamen). WT and Apc ${ }^{\mathrm{Min}}$ mice were euthanized at day 80 after birth. The colitis-associated mouse colon cancer model was induced as follows: on day 1, 6-week-old mice were intraperitoneally injected with $10 \mathrm{mg} / \mathrm{kg}$ AOM (Sigma) and maintained on a regular diet and water for 5 days. After 5 days, the mice received water supplemented with 3\% DSS (MP Biomedicals) for 5 days. Afterward, the mice were maintained on regular water for 12 days and subjected to two additional DSS treatment cycles. The AOM/DSS group and control mice were euthanized at day 80 after the first injection of AOM. For tumor growth assays, $5 \times 10^{6}$ cells were subcutaneously injected into the lower back regions of 6-week-old male nude mice for four weeks ( $n=5$ per group). For orthotropic metastasis, 6-week-old male nude mice were anesthetized and their spleens were exteriorized by laparotomy, and then $5 \times 10^{5}$ cells were injected into the spleens for four weeks ( $n=5$ per group). Tumor growth and hepatic and intestinal metastases at day 28 were monitored using the live animal Lumina II system (Xenogen IVIS system).

\section{Cell culture}

The human embryonic kidney epithelial cell line 293T and the CRC cell lines SW620 and HCT116 were provided by Dr Han You (State Key Laboratory of Cellular Stress Biology, School of Life Sciences, Xiamen University, Xiamen). LS174t, HT29, Caco2 and LoVo cells were obtained from the Institute of Biochemistry and Cell Biology, Chinese Academy of Sciences, Shanghai. HCT116 cells were maintained in McCoy's 5A media supplemented with 10\% fetal bovine serum. 293T, SW620, LS174t and HT29 cells were cultured in DMEM supplemented with $10 \%$ fetal bovine serum. Caco2 and LoVo cells were maintained in RPMI1640 media supplemented with 10\% fetal bovine serum.

\section{Plasmid construction and generation of stable cell lines}

Plasmid construction was performed as described previously [38]. For the generation of stable miR543-overexpressing cell lines, a lentivirus-mediated packaging system containing four plasmids- $\mathrm{pCDH}-$ miR-543 or control plasmid (scrambled miRNA), pMDL, REV and VSVG-was used. For stable knockdown of miR-543 in HCT116 cells, pLL3.7-puro containing the best hairpin sequence targeting miR-543 (GCGGTGCACTTCTTTTTCA) or a control plasmid (control hairpin) was co-transfected with pMDL, REV and VSVG. The transfection and lentiviral infection processes were similar to those described previously [39]. To generate miRNA-insensitive KRAS, MTA1 and HMGA2 constructs, regions of human KRAS CDS (NM_033360.3) at 193-762 bp, MTA1 CDS (NM_004689.3) at 188-2335 bp and HMGA2 CDS (NM_003483.4) at 812-1141 bp were generated by PCR amplification and then subcloned into the pCMV-5 plasmid (Addgene). The primers used to generate these constructs are listed in Supplementary Table S1.

\section{Quantitative real-time PCR}

Total RNA was prepared with the Trizol reagent (Invitrogen) according to the manufacturer's instructions. For miRNA reverse transcription, cDNA was synthesized using TaqManH MicroRNA Reverse Transcription Kit (ABI) with $100 \mathrm{ng}$ total RNA. For mRNA reverse transcription, cDNA was synthesized using ReverTra AceH qPCR RT Kit (TOYOBO) with $1 \mathrm{mg}$ total RNA. Real-time PCRs were performed using SYBRH Select Master Mix for CFX (Invitrogen). Relative quantification was achieved by normalization to the amount of GAPDH (for mRNAs) or snRNA U6 (for miRNAs). The $2^{-\Delta \Delta \mathrm{Ct}}(\Delta \mathrm{Ct}=\mathrm{CtmiR}-543-$ CtU6) method for quantitation of gene expression was used to determine miR-543 relative expression levels. The $\Delta \Delta \mathrm{Ct}$ was calculated by subtracting the $\Delta \mathrm{Ct}$ of the reference sample (a normal clinical or mouse tissue sample was chosen for the normalization of clinical samples or samples in two 
mouse models, LoVo cell was chosen for the normalization of five $\mathrm{CRC}$ cell lines) from the $\Delta \mathrm{Ct}$ of each sample. The mean relative miR-543 expression (5.8) of all clinical tumor samples was chosen as the cut-off to classify a tumor sample was High or Low according to their miR-543 expression level. The primers used are shown in Supplementary Table S2.

\section{Western blotting}

Western blotting was performed as described previously [38]. Cell lysates were subjected to SDSpolyacrylamide gel and immunoblot analysis with antibodies against the following proteins: KRAS and HMGA2 (GeneTex); Vimentin (R \& D); Cyclin D1 (Millipore); LOX (Abcam); p-FAK (Invitrogen); E-Cadherin, N-Cadherin and b-catenin (BD); MTA1, STAT3, ERK, p-ERK, MEK and p-MEK (Cell Signaling); b-actin (Sigma Aldrich).

\section{Cell migration and invasion assays}

Migration and invasion assays were performed using Transwell plates (Corning) with $8 \mu \mathrm{m}$-pore size membranes without Matrigel (for migration assays) or with Matrigel (for invasion assays). For migration assays, $3 \times 10^{4} \mathrm{SW620}$, $3 \times 10^{4} \mathrm{LoVo}, 5 \times 10^{4} \mathrm{HCT} 116$ cells or the same number of corresponding miR-543-overexpressing or miR-543knockdown cells were seeded in the top chambers. For the Matrigel-coated membrane Transwell invasion assays, $8 \times 10^{4} \mathrm{SW} 620,8 \times 10^{4} \mathrm{LoVo}, 10 \times 10^{4} \mathrm{HCT} 116$ cells or the same number of corresponding miR-543-overexpressing or miR-543-knockdown cells were seeded in the top chambers of Transwell plates. The migrating or invading cells were counted and photographed.

\section{miRNA reporter luciferase assay}

293 T or SW620 cells were seeded into a 24-well plate and cotransfected with miR-543 or control and 3'UTR-luciferase plasmids. The cells were lysed at $48 \mathrm{~h}$ posttransfection, and the luciferase activity was measured using the Dual-Glo Luciferase Assay System (Promega) and normalized to Renilla luciferase activity.

\section{Cell proliferation and colony formation assay}

For cell proliferation assay, cells were seeded on 96 -well plates at initial density of $\left(3 \times 10^{3}\right.$ cells/well $)$. The cells were stained with $100 \mu \mathrm{l}$ sterile MTT dye $(0.5 \mathrm{mg} / \mathrm{ml}$, Sigma-Aldrich, MO) at each time point for $4 \mathrm{~h}$ at $37^{\circ} \mathrm{C}$ followed by removal of the culture medium and addition of $150 \mu \mathrm{l}$ of dimethyl sulphoxide. The absorbance was measured at $490 \mathrm{~nm}$. All experiments were performed in triplicates. For colony formation assay, cells were seeded in six-well plates at a density of 300 cells/well and maintained in medium for 12-20 days. The cells were washed with PBS, fixed in methanol for $15 \mathrm{~min}$ and stained with crystal violet for $15 \mathrm{~min}$. The plates were then photographed, and the colonies were counted. At least three independent experiments were carried out for each assay.

\section{Gelatin zymography}

Cells were plated onto $6 \mathrm{~cm}$ culture plates and were incubated with medium that did not contain $\mathrm{FBS}$ at $37^{\circ} \mathrm{C}$. After $24 \mathrm{~h}$, the medium collected from the incubated cells was concentrated and mixed with substrate gel sample buffer and then loaded without boiling onto 8\% SDS polyacrylamide gel that contained $1 \%$ gelatin (Sigma, St. Louis, MO). After performing electrophoresis, the gel was then soaked in $2 \%$ Triton X-100 with gentle shaking for 60 min with single change of detergent solution. The gel was rinsed and next incubated for 1 day in substrate buffer (50 mM Tris- $\mathrm{HCl}, \mathrm{pH} 7.5,5 \mathrm{mM} \mathrm{CaCl}_{2}$, and $0.02 \%$ $\mathrm{NaN}_{3}$ ). Following the incubation, the gel was stained with $0.05 \%$ Coomassie brilliant blue G-250 and then destained in $10 \%$ acetic acid and $20 \%$ methanol.

\section{Immunohistochemical staining}

Tissue sections from specimens of primary colorectal cancer were fixed in 10\% formaldehyde and embedded in paraffin. Sections were then cut and stained using immunohistochemistry as previously described [39]. Briefly, paraffin-embedded tissue sections were processed for antigen retrieval by heating the sections in $10 \mathrm{mM}$ sodium citrate $(\mathrm{pH} 6.0)$ at $95^{\circ} \mathrm{C}$ for $20 \mathrm{~min}$. Sections were immunostained with antibodies anti-KRAS (1:200), anti-MTA1 (1:200) or anti-HMGA2 (1:200). The immunostaining was performed with an ABC staining system (Santa Cruz Biotechnology, Santa Cruz, CA) using an avidin-biotinylated-peroxidase detection method. For quantitation, average integrated optical density (IOD) was obtained by analyzing five fields in each slide evaluated by Image-Pro Plus software (version 6.0) for immunohistochemical staining of KRAS, MTA1 and HMGA2. The average IOD of tumor tissue was divided by the average IOD of paired normal tissue to get the relative average IOD.

\section{Statistical analysis}

All quantitative data are expressed as the mean \pm SD. Significant differences were analyzed using Student's $t$-test to compare two groups of independent samples. $p$ values of 0.05 or less were considered as significant.

\section{ACKNOWLEDGMENTS AND FUNDING}

This work was supported by grants from the National Nature Science Foundation of China (No. 81572598, 81372841, 31171339), the Fundamental Research Funds for the Central Universities (20720150060), the National Science Foundation of China for Fostering Talents in Basic Research (No. J1310027), Key Project of Science and Technology Project of Fujian Province (No. 2014D017) 
and the Science and Technology Project of Natural Science Foundation of Fujian Province (No. 2015J01564).

\section{CONFLICTS OF INTEREST}

The authors declare no conflicts of interest.

\section{REFERENCES}

1. Chen W, Zheng R, Zeng H, Zhang S, He J. Annual report on status of cancer in China, 2011. Chin J Cancer Res. 2015; 27:2-12.

2. Bao S, Ouyang G, Bai X, Huang Z, Ma C, Liu M, Shao R, Anderson RM, Rich JN, Wang XF. Periostin potently promotes metastatic growth of colon cancer by augmenting cell survival via the Akt/PKB pathway. Cancer Cell. 2004; 5:329-339.

3. Ebi H, Corcoran RB, Singh A, Chen Z, Song Y, Lifshits E, Ryan DP, Meyerhardt JA, Benes C, Settleman J, Wong KK, Cantley LC, Engelman JA. Receptor tyrosine kinases exert dominant control over PI3K signaling in human KRAS mutant colorectal cancers. J Clin Invest. 2011; 121:431143-21.

4. Donnard E, Asprino PF, Correa BR, Bettoni F, Koyama FC, Navarro FC, Perez RO, Mariadason J, Sieber OM, Strausberg RL, Simpson AJ, Jardim DL, Reis LF, et al. Mutational analysis of genes coding for cell surface proteins in colorectal cancer cell lines reveal novel altered pathways, druggable mutations and mutated epitopes for targeted therapy. Oncotarget. 2014;5:9199-9213. doi: 10.18632/oncotarget.2374.

5. Ling H, Pickard K, Ivan C, Isella C, Ikuo K, Mitter R, Spizzo R, Bullock MD, Braicu C, Pileczki V, Vincent K, Pichler M, Stiegelbauer V, et al. The clinical and biological significance of MIR-224 expression in colorectal cancer metastasis. Gut. 2015; pii: gutjnl-2015-309372.

6. Walther A, Johnstone E, Swanton C, Midgley R, Tomlinson I, Kerr D. Genetic prognostic and predictive markers in colorectal cancer. Nat Rev Cancer. 2009; 9:489-499.

7. Bronte G, Silvestris N, Castiglia M, Galvano A, Passiglia F, Sortino G, Cicero G, Rolfo C, Peeters M, Bazan V, Fanale D, Giordano A, Russo A. New findings on primary and acquired resistance to anti-EGFR therapy in metastatic colorectal cancer: do all roads lead to RAS? Oncotarget. 2015; 6:24780-24796. doi: 10.18632/oncotarget.4959.

8. Dow LE, O'Rourke KP, Simon J, Tschaharhaneh DF, Es J, Clevers H, Lowe SW. Apc restoration promotes cellular differentiation and reestablishes crypt homeostasis in colorectal cancer. Cell. 2015; 161:1539-1552.

9. Yu J, Wu WK, Li X, He J, Li XX, Ng S, Yu C, Gao Z, Yang J, Li M, Wang Q, Liang Q, Pan Y, et al. Novel recurrently mutated genes and a prognostic mutation signature in colorectal cancer. Gut. 2015; 64:636-645.

10. Goel A, Boland CR. Epigenetics of colorectal cancer. Gastroenterology. 2012; 143:1442-1460.e1.

11. Bartel DP. MicroRNAs: genomics, biogenesis, mechanism, and function. Cell. 2004; 116:281-297.
12. Calin GA, Croce CM. MicroRNA signatures in human cancers. Nat Rev Cancer. 2006; 6:857-866.

13. Krijger Id, Mekenkamp LJ, Punt CJ, Nagtegaal ID. MicroRNAs in colorectal cancer metastasis. J Pathol. 2011; 224:438-447.

14. Haga CL, Phinney DG. MicroRNAs in the imprinted DLK1-DIO3 region repress the epithelial-to-mesenchymal transition by targeting the TWIST1 protein signaling network. J Biol Chem. 2012; 287:42695-42707.

15. Bing L, Hong C, Li-Xin S, Wei G. MicroRNA-543 suppresses endometrial cancer oncogenicity via targeting FAK and TWIST1 expression. Arch Gynecol Obstet. 2014; 290:533-541.

16. Yu L, Zhou L, Cheng Y. MicroRNA-543 acts as an oncogene by targeting PAQR3 in hepatocellular carcinoma. Am J Cancer Res. 2014; 4:897-906.

17. Moser AR, Luongo C, Gould KA, McNeley MK, Shoemaker AR, Dove WF. ApcMin: a mouse model for intestinal and mammary tumorigenesis. Eur J Cancer. 1995; 31A:1061-1064.

18. Grivennikov S, Karin E, Terzic J, Mucida D, Yu GY, Vallabhapurapu S, Scheller J, Rose-John S, Cheroutre H, Eckmann L, Karin M. IL-6 and Stat3 are required for survival of intestinal epithelial cells and development of colitisassociated cancer. Cancer Cell 2009; 15:103-113.

19. Neufert C, Becker C, Türeci Ö, Waldner MJ, Backert I, Flooh K, Atreya I, Leppkes M, Jefremow A, Vieth M, Schneider-Stock R, Klinger P, Greten FR, et al. Tumor fibroblast-derived epiregulin promotes growth of colitisassociated neoplasms through ERK. J Clin Invest. 2013; 123:1428-1443.

20. Fang JY, Richardson BC. The MAPK signalling pathways and colorectal cancer. Lancet Oncol. 2005; 6:322-327.

21. Liao WT, Ye YP, Zhang NJ, Li TT, Wang SY, Cui YM, Qi L, Wu P, Jiao HL, Xie YJ, Zhang C, Wang JX, Ding YQ. MicroRNA-30b functions as a tumour suppressor in human colorectal cancer by targeting KRAS, PIK3CD and BCL2. J Pathol. 2014; 232:415-427.

22. Zhu W, Cai MY, Tong ZT, Dong SS, Mai SJ, Lia YJ, Bian XW, Lin MC, Kung HF, Zeng YX, Guan XY, Xie D. Overexpression of EIF5A2 promotes colorectal carcinoma cell aggressiveness by upregulating MTA1 through C-myc to induce epithelialmesenchymal transition. Gut. 2012; 61: 562-575.

23. Morishita A, Zaidi MR, Mitoro A, Sankarasharma D, Szabolcs M, Okada Y, D'Armiento J, Chada K. HMGA2 is a driver of tumor metastasis. Cancer Res. 2013; 73:4289-4299.

24. Pakala SB, Rayala SK, Wang RA, Ohshiro K, Mudvari P, Reddy SD, Zheng Y, Pires R, Casimiro S, Pillai MR, Costa L, Kumar R. MTA1 promotes STAT3 transcription and pulmonary metastasis in breast cancer. Cancer Res. 2013; 73:3761-3770.

25. Kessenbrock K, Plaks V, Werb Z. Matrix metalloproteinases: regulators of the tumor microenvironment. Cell. 2010; 141: $52-67$. 
26. Sun M, Gomes $\mathrm{S}$, Chen P, Frankenberger CA, Sankarasharma D, Chuang CH, Chada KK, Rosner MR. RKIP and HMGA2 regulate breast tumor survival and metastasis through lysyl oxidase and syndecan-2. Oncogene. 2014; 33:3528-3537.

27. Payne SL, Fogelgren B, Hess AR, Seftor EA, Wiley EL, Fong SF, Csiszar K, Hendrix MJ, Kirschmann DA. Lysyl oxidase regulates breast cancer cell migration and adhesion through a hydrogen peroxide-mediated mechanism. Cancer Res. 2005; 65:11429-11436.

28. Loo JM, Scherl A, Nguyen A, Man F, Weinberg E, Zeng Z, Saltz L, Paty PB, Tavazoie SF. Extracellular metabolic energetics can promote cancer progression. Cell. 2015; 160:393-406.

29. Morikawa K, Walker SM, Nakajima M, Pathak S, Jessuo JM, Fidler IJ. Influence of organ environment on the growth, selection, and metastasis of human colon carcinoma cells in nude mice. Cancer Res. 1988; 48:6863-6871.

30. Nguyen DX, Bos PD, Massague J. Metastasis: from dissemination to organ-specific colonization. Nat Rev Cancer. 2009; 9:274-284.

31. Scherr M, Venturini L, Battmer K, Schoenitz MS, Schaefer D, Dallmann I, Ganser A, Eder M. Lentivirus-mediated antagomir expression for specific inhibition of miRNA function. Nucleic Acids Res. 2007; 35:e149.

32. Bos JL, Fearon ER, Hamilton SR, Vries MV, Boom JH, van der Eb AJ, Vogelstein B. Prevalence of ras gene mutations in human colorectal cancers. Nature. 1987; 327:293-297.
33. Toh Y, Nicolson GL. The role of the MTA family and their encoded proteins in human cancers: molecular functions and clinical implications. Clin Exp Metastasis. 2009; 26:215-227.

34. Levy DE, Darnell JE Jr. Stats: transcriptional control and biological impact. Nat Rev Mol Cell Biol. 2002; 3:651-662.

35. Yu H, Pardoll D, Jove R. STATs in cancer inflammation and immunity: a leading role for STAT3. Nat Rev Cancer. 2009; 9:798-809.

36. Wang $\mathrm{X}$, Liu X, Li A, Chen L, Lai L, Lin H, Hu S, Yao L, Peng J, Loera S, Xue L, Zhou B, Zhou L, et al. Overexpression of HMGA2 promotes metastasis and impacts survival of colorectal cancers. Clin Cancer Res. 2011;17:2570-2580.

37. Croce CM. Causes and consequences of microRNA dysregulation in cancer. Nat Rev Genet. 2009; 10:704-714.

38. Lin Y, Liu AY, Fan C, Zheng H, Li Y, Zhang C, Wu S, Yu D, Huang Z, Liu F, Luo Q, Yang CJ, Ouyang G. MicroRNA$33 \mathrm{~b}$ inhibits breast cancer metastasis by targeting HMGA2, SALL4 and Twist1. Sci Rep. 2015; 5:9995.

39. Fang X, Cai Y, Liu J, Wang Z, Wu Q, Zhang Z, Yang CJ, Yuan L, Ouyang G. Twist 2 contributes to breast cancer progression by promoting an epithelial-mesenchymal transition and cancer stem-like cell self-renewal. Oncogene. 2011; 30:4707-4720. 